\title{
Anti-methicillin Resistant Staphylococcus aureus (MRSA) Compounds Isolated from Laurus nobilis
}

\author{
Nao Otsuka, ${ }^{a}$ Mei-Hua Liu, ${ }^{a}$ Sumiko Shiota, ${ }^{b}$ Wakano Ogawa, ${ }^{a}$ Teruo Kuroda, ${ }^{a}$ \\ Tsutomu Hatano, ${ }^{c}$ and Tomofusa TsuchiYA ${ }^{*} a$ \\ ${ }^{a}$ Department of Molecular Microbiology, Graduate School of Medicine, Dentistry and Pharmaceutical Sciences, Okayama \\ University; ' Department of Pharmacognosy, Graduate School of Medicine, Dentistry and Pharmaceutical Sciences, \\ Okayama University; Tsushima, Okayama 700-8530, Japan: and ${ }^{b}$ Laboratory of Pathogenic Microbiology, School of \\ Pharmacy, Shujitsu University; Nishigawara, Okayama 703-8516, Japan.
}

Received March 7, 2008; accepted June 13, 2008; published online June 23, 2008

We found that an extract from Laurus nobilis L. (Lauraceae) leaves showed antibacterial activity against methicillin-resistant Staphylococcus aureus (MRSA). We purified two flavonoids as the effective compounds and identified them as kaempferol 3-O- $\alpha$-L-(2", 4 "-di- $E$-p-coumaroyl)-rhamnoside (C2) and kaempferol 3-O- $\alpha$-L-(2"-Zp-coumaroyl-4"-E-p-coumaroyl)-rhamnoside (C3). Both compounds showed strong antibacterial activity not only against MRSA but also against vancomycin-resistant enterococci (VRE). There was low or no antibacterial activity of C2 and C3 for Streptococcus pneumoniae, Pseudomonas aeruginosa and Serratia marcescens.

Key words methicillin-resistant Staphylococcus aureus; Laurus nobilis; vancomycin-resistant enterococci; anti-methicillin-resistant Staphylococcus aureus compound; anti-vancomycin-resistant enterococci

The incidence of infectious disease caused by drug resistant bacteria that are represented by methicillin-resistant Staphylococcus aureus (MRSA), vancomycin-resistant enterococci (VRE) and so on is extremely important in both community and hospital settings. ${ }^{1}$ Methicillin was introduced in Europe in 1959 and in the United States in 1961, and the first cases of MRSA were reported in the United Kingdom in 1961, followed soon thereafter by reports in Japan and Australia. ${ }^{1)}$ So far, tremendous numbers of the MRSA strain have been reported in the world. Aucken et al. reported an epidemic comprised of MRSA, designated EMRSA-17, that showed resistances to multiple antibacterial agents including methicillin, ciprofloxacin, erythromycin, fusidic acid, rifampicin, gentamicin, kanamycin, neomycin, streptomycin and tetracycline. ${ }^{2)}$ In general, MRSA strains show resistance against multiple antimicrobial agents although the ranges and extents of these resistances are versatile. In many years past, vancomycin was the only effective drug for serious MRSA infections. In 1996, $S$. aureus with intermediate resistance to vancomycin (VISA; MIC was 8 to $16 \mu \mathrm{g} / \mathrm{ml}$ ) was first observed in a strain isolated from a hospitalized patient in Japan. ${ }^{3)}$ Moreover, in 2002, a high-level vancomycin resistant S. aureus (VRSA: MIC was $1024 \mu \mathrm{g} / \mathrm{ml}$ ) strain was isolated from a dialysis patient in the U.S.A. ${ }^{4)}$ The report provided a genetic analysis in that the vanA gene made MRSA highly resistant to vancomycin. Up to the present day, four new agents with anti-MRSA activity have been introduced (quinupristin-dalfopristin, linezolid, daptomycin and tigecycline) in the U.S.A. But among them, only quinupristin-dalfopristin and linezolid are clinically available in Japan. As was concerned, resistance to linezolid has already emerged in clinical isolates of $S$. aureus. ${ }^{5,6)}$

VRE was not observed until the 1980s. During the 1990s, however, a drastic rise in VRE infections occurred. It has been reported that approximately $30 \%$ of all enterococci isolated from intensive care patients units are now resistant to vancomycin. ${ }^{7)}$ Among the clinically isolated enterococcal species, 80-90\% are Enterococcus faecalis and others are mainly E. faecium. ${ }^{8)}$ Intrinsically, enterococci (especially $E$. faecium) possess a broad range of resistance against antimicrobials.

Under these circumstances, the demand for the development of new antibacterial agents effective against MRSA and/or VRE is a matter of great urgency. Thus, we have been trying to discover effective growth inhibitors against MRSA and/or VRE. There are several reports on natural anti-MRSA compounds, such as 3-arylcoumarines, ${ }^{9)}$ licoricidin, ${ }^{10)}$ cudraxanthone $\mathrm{S},{ }^{11)}$ piperitylmagnolol, ${ }^{12)}$ and isolupalbigenin. ${ }^{13)}$ Here we report two anti-MRSA compounds, which showed stronger anti-MRSA activity than these compounds, isolated from leaves of Laurus nobilis (laurel), a kind of herb.

\section{MATERIALS AND METHODS}

Materials MRSA strains OM481, OM505, OM584, OM623 and N315 are clinically isolated strains. MRSA COL and MSSA (methicillin-sensitive $S$. aureus) 209P are laboratory collections. Leaves of L. nobilis (laurel) cultivated in Turkey were purchased from Toho TH2, Inc. (Kobe, Japan). Ciprofloxacin, erythromycin, norfloxacin oxacillin, tetracycline, vancomycin, isoquercitrin, kaempferol, $p$-coumaric acid, quercetin, quercitrin and rutin were from commercial sources.

Extraction and Purification of Effective Compounds Dried ground leaves $(1.5 \mathrm{~kg})$ of $L$. nobilis were homogenized in $70 \%$ acetone and filtered. The acetone extract was then concentrated and extracted with hexane, ethyl acetate, successively. The ethyl acetate extract ( $45 \mathrm{~g}$ ) was subjected to column chromatography over DIAION HP-20 (Mitsubishi Kasei Co., Ltd.) and eluted with $\mathrm{H}_{2} \mathrm{O}$ and aqueous methanol $(60,70,80,90,100 \%)$ in a stepwise gradient manner. Thereafter, a $90 \%$ methanol fraction $(3.5 \mathrm{~g})$ which showed the highest anti-MRSA activity was subjected to a Sephadex LH20 (Amersham Biosciences, Sweden) column, eluted with $100 \%$ ethanol, and fractionated into 8 fractions (Fraction A to $\mathrm{H})$. The Fraction D (1.6 g) which showed the highest activ- 
ity was subjected to preparative TLC using Kieselgel 60F254 plates $(0.2 \mathrm{~mm}$ thick, Merck) and developed with a $\mathrm{CHCl}_{3}-\mathrm{MeOH}-\mathrm{HCOOH}(9: 1: 1)$ solvent system. A zone showing the highest activity was collected $(120 \mathrm{mg})$. The preparative HPLC was performed on an ODS-3 inertsil column (GL Science Inc.), eluted with $\mathrm{MeOH}-\mathrm{H}_{2} \mathrm{O}(70: 30)$ and effective compounds $\mathrm{C} 2(27.4 \mathrm{mg})$ and $\mathrm{C} 3(62.4 \mathrm{mg})$ were obtained.

Susceptibility Testing Minimum inhibitory concentrations (MICs) of antimicrobial agents were determined by broth dilution techniques, according to the instructions of the Clinical and Laboratory Standards Institute (CLSI; formerly NCCLS). The MIC determinations were made in triplicate on separate occasions. Broth $\mathrm{MIC}$ testing was performed in 96-well microtiter trays with an inoculum of about $10^{5} \mathrm{CFU}$ in $100 \mu \mathrm{l}$ of Mueller-Hinton broth (Difco) supplemented with $0.85 \% \mathrm{NaCl}$. MIC values were obtained after incubation at $35^{\circ} \mathrm{C}$ for $24 \mathrm{~h}$. Compounds $\mathrm{C} 2$ and $\mathrm{C} 3$ were dissolved in $\mathrm{MeOH}$ before dilution into $\mathrm{MHB}$ for MIC determinations.

\section{RESULTS}

Purification and Identification of Effective Compounds We prepared methanol extracts from many plants and tested the antibacterial activities of these extracts. We found that an extract from the leaves of Laurus nobilis (laurel) showed strong anti-MRSA activity. We also found that the extract showed potent anti-VRE activity. We tried to purify the antiMRSA compound(s) by using column chromatography with DIAION HP-20, Sephadex LH-20, thin-layer chromatography (TLC) and HPLC as described under Materials and Methods. We obtained two effective compounds, and identified them as kaempferol 3-O- $\alpha$-L-(2", $4^{\prime \prime}-$ di- $E$ - $p$-coumaroyl)rhamnoside (C2) and kaempferol 3-O- $\alpha-\mathrm{L}-\left(2^{\prime \prime}-Z-p\right.$-coumaroyl-4"-E-p-coumaroyl)-rhamnoside (C3) (Fig. 1) by using ${ }^{1} \mathrm{H}$ - and ${ }^{13} \mathrm{C}-\mathrm{NMR}$, UV-visible spectra, ESI-MS spectra, optical rotation.

Anti-MRSA Activities of C2 and C3 We measured the MICs of C2 and C3 with several MRSA strains. We observed MIC values of 1 to $2 \mu \mathrm{g} / \mathrm{ml}$ with the MRSA strains tested (Table 1). S. aureus OM481, OM505, OM584 and OM623 are clinically isolated MRSA strains that showed very high MICs of oxacillin, fluoroquinolones and erythromycin. Thus, these antimicrobial agents are basically ineffective on these MRSA strains. The MICs of these drugs and of tetracycline with COL and N315 MRSA strains were diverse. C2 and C3 were able to inhibit the growth of all of these MRSA strains at relatively low concentrations. MICs of C2 and C3 with these MRSA strains were a little higher than that of vancomycin (Table 1). The $\mathrm{C} 2$ and $\mathrm{C} 3$ compounds showed a similar but slightly lower MIC $(0.5 \mu \mathrm{g} / \mathrm{ml})$ with a methicillinsensitive S. aureus 209P, an MSSA strain, than with the MRSA strains. Thus, C2 and C3 are effective on both MRSA and MSSA at similar concentrations.

Structure-Activity Relationship We tested the effects of compounds with structures similar or partially similar to the structures of $\mathrm{C} 2$ and $\mathrm{C} 3$ on MRSA and MSSA to get some insights into the structure-activity relationship. The $\mathrm{C} 2$ and C3 compounds consist of kaempferol, rhamnose, and coumaroyl moieties. Kaempferol and $p$-coumaric acid did not show anti-MRSA and anti-MSSA activities (Table 1), and rhamnose showed no growth inhibition (data not shown).
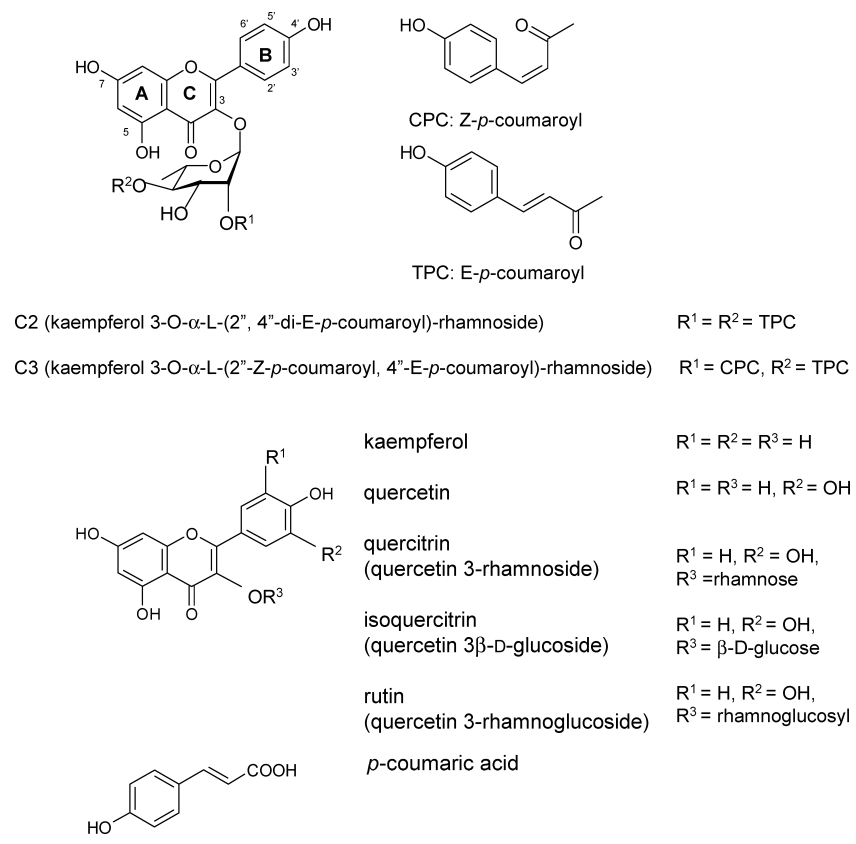

Fig. 1. Structures of $\mathrm{C} 2, \mathrm{C} 3$, and Related Compounds

Table 1. Antibacterial Activity of $\mathrm{C} 2$ and $\mathrm{C} 3$ on $S$. aureus

\begin{tabular}{|c|c|c|c|c|c|c|c|}
\hline \multirow{2}{*}{ Compound } & \multicolumn{7}{|c|}{$\mathrm{MIC}(\mu \mathrm{g} / \mathrm{ml})$} \\
\hline & OM481 & OM505 & OM584 & OM623 & $\mathrm{COL}$ & N315 & 209P \\
\hline $\mathrm{C} 2$ & 1 & 1 & 2 & 2 & 1 & 1 & 0.5 \\
\hline $\mathrm{C} 3$ & 1 & 2 & 2 & 2 & 1 & 1 & 0.5 \\
\hline Kaempferol & $>256$ & $>256$ & $>25 \overline{6}$ & $>256$ & $>256$ & $>256$ & $>256$ \\
\hline Quercetin & $>256$ & $>256$ & $>256$ & $>256$ & $>256$ & $>256$ & $>256$ \\
\hline Quercitrin & $>256$ & $>256$ & $>256$ & $>256$ & $>256$ & $>256$ & $>256$ \\
\hline Isoquercitrin & $>256$ & $>256$ & $>256$ & $>256$ & $>256$ & $>256$ & $>256$ \\
\hline Rutin & $>256$ & $>256$ & $>256$ & $>256$ & $>256$ & $>256$ & $>256$ \\
\hline$p$-Coumaric acid & $>256$ & $>256$ & $>256$ & $>256$ & $>256$ & $>256$ & $>256$ \\
\hline Oxacillin & 512 & 128 & 256 & 256 & 512 & 8 & 0.13 \\
\hline Ciprofloxacin & 8 & 1 & 16 & 8 & $<0.13$ & 0.25 & $<0.13$ \\
\hline Norfloxacin & 128 & 8 & 64 & 64 & 1 & 2 & 0.5 \\
\hline Erythromycin & $>1024$ & $>128$ & $>1024$ & $>128$ & 0.13 & $>1024$ & 2 \\
\hline Tetracycline & 4 & 0.25 & 128 & 64 & 128 & 0.13 & 0.13 \\
\hline Vancomycin & 0.25 & 0.25 & 0.25 & 0.25 & 0.25 & 0.25 & $<0.25$ \\
\hline
\end{tabular}


Table 2. Antibacterial Activity of $\mathrm{C} 2$ and $\mathrm{C} 3$

\begin{tabular}{lrcccc}
\hline \hline & & & & MIC $(\mu \mathrm{g} / \mathrm{ml})$ \\
\cline { 2 - 6 } Compound & $\begin{array}{c}\text { E. faecium } \\
\text { FN-1 }\end{array}$ & $\begin{array}{c}\text { E. faecalis } \\
\text { NCTC12201 }\end{array}$ & $\begin{array}{c}\text { S. pneumoniae } \\
\text { R6 }\end{array}$ & $\begin{array}{c}\text { P. aeruginosa } \\
\text { PAO1 }\end{array}$ & $\begin{array}{c}\text { S. marcescens } \\
\text { NUSM8906 }\end{array}$ \\
\hline C2 & 8 & 4 & $>16$ & $>16$ & $>16$ \\
C3 & 8 & 4 & $>16$ & $>128$ & $>16$ \\
Vancomycin & $>128$ & $>128$ & $<0.25$ & $>256$ & $>128$ \\
Kaempferol & $>256$ & $>256$ & 256 & $>256$ & \\
\hline
\end{tabular}

Thus, each one of these compounds alone is not enough for the inhibition of MRSA growth. Compounds that possess structures similar to $\mathrm{C} 2$ and $\mathrm{C} 3$, i.e., quercetin, quercitrin (quercetin 3-rhamnoside), isoquercetin (quercetin $3 \beta$-D-glucoside) and rutin (quercetin 3-rhamnoglucoside) (Fig. 1), showed no detectable anti-MRSA and anti-MSSA activities (Table 1). Thus, it seems at the present time that the intact structures of $\mathrm{C} 2$ and $\mathrm{C} 3$ are important for antibacterial activity.

Antibacterial Activity on Some Other Bacteria We also tested the antibacterial activities of $\mathrm{C} 2$ and $\mathrm{C} 3$ on some other bacteria such as E. faecium $\mathrm{FN}-1, E$. faecalis NCTC12210, Streptococcus pneumoniae R6, Pseudomonas aeruginosa PAO1 and Serratia marcescens NUSM8906. E. faecium, E. faecalis and S. pneumoniae are Gram-positive bacteria, and $P$. aeruginos $a$ and $S$. marcescens are Gram-negative bacteria. We observed MIC values being 8 and $4 \mu \mathrm{g} / \mathrm{ml}$ for $E$. faecium $\mathrm{FN}-1$ and $E$. faecalis NCTC12201, respectively, with both $\mathrm{C} 2$ and $\mathrm{C} 3$ (Table 2). Since E. faecium $\mathrm{FN}-1$ and E. faecalis NCTC12201 are VRE strains, the MIC of vancomycin with these strains was very high, higher than $128 \mu \mathrm{g} / \mathrm{ml}$ (Table 2). Thus, C2 and C3 are moderately effective on the VRE strains tested. On the other hand, the MIC value of C2 and C3 were higher than $16 \mu \mathrm{g} / \mathrm{ml}$ for $S$. pneumoniae, $P$. aeruginosa and $S$. marcescens. Kaempferol was not effective on all of these bacteria (Table 2).

\section{DISCUSSION}

We isolated anti-MRSA compounds from L. nobilis (laurel) leaves and identified them as $\mathrm{C} 2$ and $\mathrm{C} 3$ (Fig. 1). Laurel is an evergreen tree widespread in the Mediterranean area and Europe, and is used as a folk medicine. Decoction or tea of leaves is often used as a carminative, an intestinal and gastric antispasmodic, an anti-diarrheal agent, for treating rheumatic pain, in treating diseases of the respiratory tract, as a cough sedative, and treating asthma and cardiac diseases. ${ }^{14}$ ) This means that the laurel contains many medicinal compounds. It seems that $\mathrm{C} 2$ and $\mathrm{C} 3$ are two such medicinal compounds in laurel. The $\mathrm{C} 2$ and $\mathrm{C} 3$ compounds belong to the flavonoids. Many research groups have so far isolated and determined the structures of flavonoids that possess antibacterial activity, or quantified the activity of commercially available flavonoids. ${ }^{15}$ ) The MIC values of $\mathrm{C} 2$ and $\mathrm{C} 3$ with MRSA are at the level of $\mu \mathrm{g} / \mathrm{ml}$. These values are in the lowest MIC value range against bacteria among flavonoids. In other words, $\mathrm{C} 2$ and $\mathrm{C} 3$ possess very strong antibacterial activity among the flavonoids. The anti-MRSA activity of $\mathrm{C} 2$ and $\mathrm{C} 3$ was much higher than those of chemotherapeutics such as oxacillin, ciprofloxacin, norfloxacin, erythromycin and tetracycline (Table 1). The activity was a little weaker than that of vancomycin, the clinical first-choice drug for MRSA infections. It should be pointed out that the $\mathrm{C} 2$ and C3 compounds are moderately effective on VRE (Table 2), of which vancomycin is ineffective. Thus, C2 and C3 could be seeds for anti-VRE agents.

The structure-activity relationship for the flavanones, depicted from their MIC measurement, has been reported with MRSA. ${ }^{16)}$ The study indicated that 5,7-dihydroxylation of the A ring and $2^{\prime}, 4^{\prime}$ - or $2^{\prime}, 6^{\prime}$-dihydroxylation of the $\mathrm{B}$ ring in the flavanone structure are important for significant anti-MRSA activity. The C2 and C3 carry 4'-hydroxyl substitution of the A ring, 5,7-dihydroxyl substitutions in the $\mathrm{B}$ ring (Fig. 1). Thus, structures of $\mathrm{C} 2$ and $\mathrm{C} 3$ mostly fit this structureactivity relationship. Furthermore, it has been reported that substitution with 3-O-acyl chains with $\mathrm{C}_{8}$ and $\mathrm{C}_{10}$ in the $\mathrm{C}$ ring also enhanced the anti-staphylococcal activity of flavonoids belonging to the flavan-3-ol class. ${ }^{17)}$ The $\mathrm{C} 2$ and C3 compounds possess 3-O-acylated rhamnose. The acyl (coumaroyl) group in $\mathrm{C} 2$ and $\mathrm{C} 3$ contains 10 carbon atoms. Thus, this portion would also contribute to the strong antiMRSA activity of $\mathrm{C} 2$ and $\mathrm{C} 3$. The $\mathrm{C} 2$ and $\mathrm{C} 3$ compounds showed almost the same MICs with several MRSA strains and an MSSA strain (Table 1). The $\mathrm{C} 2$ and $\mathrm{C} 3$ compounds are isomers regarding the acyl (coumaroyl) portion. This result suggests that the isomeric fine structure of this portion is not important for antibacterial activity of $\mathrm{C} 2$ and $\mathrm{C} 3$. We propose that the two acyl groups in $\mathrm{C} 2$ and $\mathrm{C} 3$ are important for the compounds to enter into MRSA cells. Similarly, 3-O-acyl chains with $\mathrm{C}_{8}$ and $\mathrm{C}_{10}$ would be important for flavan-3-ols entry into cells.

It has been reported that laurel leaves containing glycosylated flavonoids and $\mathrm{C} 2$ have been identified in Laurus nobilis ${ }^{18)}$ Ocotea vellosiana (Lauraceae) ${ }^{19)}$ and in Platanus acenifolia (Platanaceae). ${ }^{20)}$ Bloor isolated C2 from Pentachondra pumila (Epacridaceae) and reported an anti-MRSA effect. ${ }^{21)}$ However, he has not reported MIC of the compound with MRSA. Thus, it was not known how active the $\mathrm{C} 2$ was against MRSA. Recently, C3 has been identified in Cinnamomum kotoense (Lauraceae) and has been shown to possess an anti-inflammatory effect. $\left.{ }^{22}\right)$

So far, antibacterial mechanisms of various flavonoids have been investigated. The best-known antibacterial mechanisms of the flavonoids include inhibition of nucleic acid synthesis, inhibition of cytoplasmic membrane function, and inhibition of energy metabolism. ${ }^{15)}$ Glycosylated flavonoids have been reported to show effects as inhibitors of bacterial type II topoisomerases, such as DNA gyrase and topoisomerase $\mathrm{IV}^{23)}$ Ohemeng et al. reported inhibitory activity of 14 flavonoids including kaempferol against E. coli DNA 
gyrase. $^{24)}$ However, kaempferol itself did not show antiMRSA activity (Table 1). Thus, it is likely that after C2 and $\mathrm{C} 3$ get into $S$. aureus cells and that these compounds, or their cleavage product kaempferol, or kaempferol 3-O-rhamnoside, inhibit DNA gyrase and topoisomerase IV.

Acknowledgments We thank Dr. Manuel Varela of Eastern New Mexico University for critically reading the manuscript. This research was supported in part by a Grant-in-Aid for Scientific Research from the Ministry of Education, Culture, Sports, Science and Technology of Japan.

\section{REFERENCES}

1) Rice L. B., Am. J. Infect. Control, 34, S11-S19 (2006).

2) Aucken H. M., Ganner M., Murchan S., Cookson B. D., Johnson A. P., J. Antimicrob. Chemother, 50, 171-175 (2002).

3) Hiramatsu K., Hanaki H., Ino T., Yabuta K., Oguri T., Tenover F. C., J. Antimicrob. Chemother, 40, 135-136 (1997).

4) Chang S., Sievert D. M., Hageman J. C., Boulton M. L., Tenover F. C., Downes F. P., Shah S., Rudrik J. T., Pupp G. R., Brown W. J., Cardo D., Fridkin S. K., N. Engl. J. Med., 348, 1342-1347 (2003).

5) Tsiodras S., Gold H. S., Sakoulas G., Eliopoulos G. M., Wennersten C., Venkataraman L., Moellering R. C., Ferraro M. J., Lancet, 358, 207-208 (2001)

6) Toh S. M., Xiong L., Arias C. A., Villegas M. V., Lolans K., Quinn J., Mankin A. S., Mol. Microbiol., 64, 1506-1514 (2007).

7) National Nosocomial Infections Surveillance (NNIS) System Report, Am. J. Infect. Control, 32, 470-485 (2004).

8) Kariyama R., Kumon H., Nippon Rinsho, 59, 733-738 (2001).
9) Hatano T., Shintani Y., Aga Y., Shiota S., Tsuchiya T., Yoshida T., Chem. Pharm. Bull., 48, 1286-1292 (2000).

10) Fukai T., Marumo A., Kaitou K., Kanda T., Terada S., Nomura T., Fitoterapia, 73, 536-539 (2002)

11) Fukai T., Oku Y., Hou A. J., Yonekawa M., Terada S., Chem. Biodivers., 1, 1385-1390 (2004).

12) Syu W. J., Shen C. C., Lu J. J., Lee G. H., Sun C. M., Chem. Biodivers., 1, 530-537 (2004).

13) Sato M., Tanaka H., Tani N., Nagayama M., Yamaguchi R., Lett. Appl. Microbiol., 43, 243-248 (2006).

14) Dall'Acqua S., Viola G., Giorgetti M., Loi M. C., Innocenti G., Chem. Pharm. Bull., 54, 1187-1189 (2006).

15) Cushnie T. P., Lamb A. J., Int. J. Antimicrob. Agents, 26, 343-356 (2005).

16) Tsuchiya H., Sato M., Miyazaki T., Fujiwara S., Tanigaki S., Ohyama M., Tanaka T., Iinuma M., J. Ethnopharmacol., 50, 27-34 (1996).

17) Stapleton P. D., Shah S., Hamilton-Miller J. M., Hara Y., Nagaoka Y., Kumagai A., Uesato S., Taylor P. W., Int. J. Antimicrob. Agents, 24, 374-380 (2004).

18) Fiorini C., David B., Fourasté I., Vercauteren J., Phytochemistry, 47, $821-824$ (1998)

19) Garcez W. S., Yoshida M., Gottlieb O. R., Phytochemistry, 39, 815816 (1995)

20) Kaouadji M., Phytochemistry, 29, 2295-2297 (1990).

21) Bloor S. J., Phytochemistry, 38, 1033-1035 (1995).

22) Kuo Y. C., Lu C. K., Huang L. W., Kuo Y. H., Chang C., Hsu F. L., Lee T. H., Planta Med., 71, 412-415 (2005).

23) Bernard F. X., Sable S., Cameron B., Provost J., Desnottes J. F., Crouzet J., Blanche F., Antimicrob. Agents Chemother, 41, 992-998 (1997).

24) Ohemeng K. A., Schwender C. F., Fu F. P., Barrett J. F., Bioorg. Med Chem. Lett., 3, 225-230 (1993). 